\title{
Fatores de risco para mortalidade neonatal no município de Serra, Espírito Santo
}

\author{
Risk factors for neonatal mortality in the city of Serra, Espírito Santo \\ Factores de riesgo para mortalidad neonatal en la ciudad de Serra, Espírito Santo
}

\author{
Eliane de Fátima Almeida Lima', Ana Inês Sousa", Rosane Harter Griep"', Cândida Caniçali Primo' \\ ' Universidade Federal do Espírito Santo, Departamento de Enfermagem. Vitória-ES, Brasil. \\ "Universidade Federal do Rio de Janeiro, Escola de Enfermagem Anna Nery, \\ Departamento de Enfermagem de Saúde Pública. Rio de Janeiro-RJ, Brasil. \\ "I" Fundação Oswaldo Cruz, Instituto Oswaldo Cruz, Laboratório de Educação em Saúde e Ambiente. \\ Rio de Janeiro-RJ, Brasil.
}

Submissão: 04-03-2011 Aprovação: 09-10-2012

\section{RESUMO}

Objetivou-se analisar os fatores de risco associados à mortalidade neonatal no município de Serra, ES. Realizou-se estudo de coorte não concorrente, utilizando a técnica de Linkage que pareou os 32.275 nascidos vivos com 273 óbitos neonatais ocorridos no período de 2001 a 2005, utilizando-se dados do SINASC e SIM. Após os ajustes na regressão logística, os fatores associados à mortalidade foram: mães sem instrução, idade materna $<15$ anos e > 35 anos, nascer em hospital público, nenhuma consulta de pré-natal, peso ao nascer.

Descritores: Mortalidade Neonatal; Fatores de Risco; Sistemas de Informação.

\section{ABSTRACT}

The objective was to analyze the risk factors associated with neonatal mortality in the city of Serra, ES. We studied nonconcurrent cohort, using the technique of Linkage that relate the 32.275 live births with 273 neonatal deaths occurred in the period from 2001 to 2005, using data from SINASC and SIM. After adjustments in the logistic regression, factors associated with mortality were: uneducated mothers, maternal age $<15$ years and $>35$ years old, born in a public hospital, no prenatal consultation, birth weight.

Key words: Neonatal Mortality; Risk Factors; Information Systems.

\section{RESUMEN}

Objetivó-se analizar los factores de riesgo asociados a la mortalidad neonatal en la ciudad de Serra, ES. Se realizó un estudio de cohorte no concurrente, utilizando la técnica de vinculación que pareó los 32.275 nacidos vivos con 273 muertes neonatales ocurrieron en el período comprendido entre 2001 y 2005, con datos de SIM y SINASC. Después de los ajustes en la regresión logística, los factores asociados a la mortalidad fueron madres sin educación, edad materna $<15$ años y $>35$ años, nacimiento en hospital público, ninguna consulta prenatal, peso al nacer.

Palabras clave: Mortalidad Neonatal; Factores de Riesgo; Sistemas de Información. 


\section{INTRODUÇÃO}

A mortalidade infantil sempre esteve presente nas discussões sobre a avaliação da assistência materno-infantil por ser um indicador sensível às condições de vida de uma população e permitir a identificação dos fatores de risco para o primeiro ano de vida ${ }^{(1-3)}$.

No Brasil, a mortalidade infantil apresentou um forte declínio na década de 80, passando de 117 óbitos por mil nascidos vivos para 50,2; redução decorrente da queda da fecundidade, da expansão do saneamento básico e da maior cobertura das campanhas de vacinação, que influenciaram na redução das doenças infecciosas nos primeiros anos de vida ${ }^{(2)}$.

Em 2005, a mortalidade infantil, no Brasil, ainda permanecia em torno de 20 óbitos por mil nascidos vivos, índice considerado alto pela Organização Mundial de Saúde ${ }^{(4-5)}$. Em várias regiões do país a mortalidade neonatal (óbitos ocorridos entre o nascimento e os 27 dias completos de vida) se constitui no principal componente da mortalidade infantil, e o estudo dos seus possíveis fatores de risco cresce em importância, pois sabe se que, dois terços dos óbitos infantis ocorrem no período neonatal, desses $50 \%$ se dão na primeira semana e metade nas primeiras 24 horas após o nascimento ${ }^{(2-3)}$.

Nos países desenvolvidos a mortalidade neonatal é, geralmente, determinada por malformações congênitas, enquanto que nos países em desenvolvimento está relacionada principalmente aos diversos fatores de risco relacionados às condições gerais de vida e acesso aos serviços de saúde ${ }^{(6-7)}$.

O baixo peso ao nascer é considerado o principal fator de risco da mortalidade neonatal, e pode ter entre suas causas o Crescimento Intrauterino Restrito (CIUR). Dentre outras causas destacam-se o baixo nível de instrução materna, o pré-natal inadequado, estado nutricional deficiente antes da gravidez, ganho de peso insuficiente durante a gestação, idade materna (inferior a 20 anos ou superior a 35 anos), infecção geniturinária, hipertensão arterial, tabagismo ausência de cônjuge e primiparidade $^{(8-9)}$.

As desigualdades da mortalidade neonatal sinalizam chances diferentes de sobrevivência em função da falta de equidade na assistência prestada, além da presença dos fatores de risco sociais e biológicos que atuam na determinação do óbito neonatal.

O estudo dos fatores de risco para mortalidade neonatal é uma estratégia útil para detectar necessidades de saúde em diferentes subgrupos populacionais e subsidiar intervenções voltadas para a redução do risco de morte no período neonatal ${ }^{(1)}$.

O presente estudo teve como objetivo identificar os fatores de risco para a mortalidade neonatal no município da Serra, estado do Espírito Santo.

\section{METODOLOGIA}

Trata-se de um estudo de coorte não concorrente desenvolvido no município de Serra, estado do Espírito Santo, no período de 01/01/2001 a 31/12/2005. Localizado na região Sudeste, o município de Serra é um dos sete municípios que compõem a Região Metropolitana da Grande Vitória. Sua sede dista apenas $20 \mathrm{~km}$, ao norte da cidade de Vitória, capital do
Estado. O processo de urbanização promoveu transformações significativas no modo de vida, na cultura, no ambiente natural e na forma de ocupação do solo do município. Na atualidade, Serra é um dos municípios brasileiros que apresenta maior aceleração do crescimento populacional. Conta com 118 bairros e representa o terceiro município capixaba mais populoso, com aproximadamente 372.000 habitantes, com uma concentração de $99,4 \%$ da população na zona urbana. O município está na Gestão Plena da Atenção Básica ampliada. Quanto ao coeficiente de mortalidade infantil, verifica-se que houve uma meIhora, passando de 14,28/1.000 NV em 2001 para 12,98/1.000 NV em 2005 e para 13,0/1.000 NV em 2006 ${ }^{(10)}$.

Os dados do estudo foram provenientes do Sistema de Informação de Mortalidade (SIM), do Sistema de Informação de Nascidos Vivos (SINASC), utilizando-se o procedimento de linkage determinístico (manual)(6). A técnica de Linkage é utilizada para ligar informações de dois sistemas e baseia-se na "ligação" ou pareamento de dois ou mais bancos de dados independentes, que possuem variáveis comuns entre si e que, por meio de uma ou mais delas, permitem identificar os indivíduos que fazem parte dos dois bancos de dados ${ }^{(4)}$.

Para realizar o pareamento, foram utilizadas informações comuns em ambos os documentos: nome da mãe, sexo do RN, data de nascimento do RN e endereço habitual da mãe. Quando a variável nome da mãe, não era coincidente, procurava-se por nomes similares e/ou sobrenomes. A discordância aceita no momento de identificação relacionou-se a grafia não idêntica por troca de alguma letra confundível como " $Z$ " por " $\mathrm{S}$ "; "G" por "J"; "U" por "L"; "V" por "W"; "A" por "E". Para serem incluídos como pareados, a data de nascimento e o sexo do RN deveriam ser coincidentes, caso contrário seriam excluídos.

Do SIM foram identificados 301 óbitos neonatais ocorridos no período estudado. Tais óbitos foram ordenados por nome da mãe, data de nascimento e sexo do RN e esses dados foram impressos. Da mesma forma, foi ordenado o banco do SINASC e foram encontrados 32.275 nascidos vivos residentes no município da Serra.

Para cada óbito encontrado digitava-se o número da declaração de óbito (DO) no banco do SINASC. Durante o processo de pareamento dos 301 óbitos neonatais, 273 foram vinculados à sua respectiva $\mathrm{DN}$ e 28 dos óbitos não tiveram suas DNs localizadas, o que representou uma perda de $9.31 \%$ dos óbitos estudados.

A seguir, agrupou-se em um único banco de dados os registros coincidentes dos dois bancos organizados a partir do SIM e SINASC e foi criada uma nova variável com duas categorias: a primeira categoria nomeada "SIM" que se refere aos óbitos neonatais pareados a DN, e a segunda categoria "NÃO" que se refere aos sobreviventes, que não foram pareados.

Para análise da mortalidade neonatal, considerou-se como variável dependente a ocorrência de morte nos primeiros 27 dias de vida (óbito neonatal). Os fatores de risco para mortalidade neonatal foram considerados como variáveis independentes e foram hierarquizadas em três níveis de determinação: distal, intermediário e proximal.

No nível distal, foram selecionadas as variáveis socioeconômicas: idade materna, escolaridade materna e tipo de 
hospital de nascimento; no nível intermediário foram selecionadas as variáveis referentes à atenção à saúde: número de consultas no pré-natal e tipo de parto; as variáveis analisadas no nível proximal foram: sexo, idade gestacional, peso, tipo de gestação, Apgar no $1^{\circ}$ e Apgar no $5^{\circ}$ minuto.

Para testar a associação de vários fatores de risco com a mortalidade neonatal foram realizadas análises univariadas entre as variáveis independentes de cada nível de determinação e o óbito neonatal utilizando-se regressão logística. Definiu-se como categoria de referência aquela que apresentasse o menor risco para a mortalidade neonatal.

Para os ajustes das Odds Ratio (OR), a abordagem hierarquizada de entrada das variáveis foi utilizada, conforme os três níveis de determinação definidos previamente pela regressão logística multivariada agrupando-se as variáveis por bloco (distais, intermediários e proximais) para observar o ajustamento no interior do bloco. Foram selecionadas previamente para a análise de regressão logística aquelas variáveis que apresentassem significância estatística com valor de $\mathrm{p} \leq 0,20$ nas análises univariadas.

Para a composição do modelo final adotou-se o procedimento stepwise forward. Todas as associações foram consideradas estatisticamente significativas se $p \leq 0,05$. Utilizou-se o pacote estatístico Social Package Statistical Science (SPSS), versão 11.0 (2002).

Os dados utilizados foram autorizados pela Secretaria de Saúde do Estado do Espírito Santo e a pesquisa foi aprovada no Comitê de Ética em Pesquisa da Escola de Enfermagem Anna Nery, da Universidade Federal do Rio de Janeiro, sob número 094/06.

\section{RESULTADOS}

Ao analisarmos as variáveis socioeconômicas (idade materna, escolaridade materna e tipo de hospital de nascimento) e sua relação com a mortalidade neonatal, verificamos pela análise univariada que as três variáveis apresentaram associação estatisticamente significante (Tabela 1).

Utilizando-se como categoria de referência os nascidos vivos de mães com idade entre 20 a 34 anos, observou-se um risco de morte cerca de 3 vezes mais elevado $(O R=2,97$; $\mathrm{IC} 95 \%=1,2-7,30$ ) entre as mães muito jovens (menores de 15 anos); entre as mais idosas (35 anos ou mais), pôde-se observar que os nascidos vivos apresentaram um risco de óbito menor $(\mathrm{OR}=1,53$; IC95\% = 1,04-2,25).

Em relação à escolaridade materna, identificou-se risco elevado de óbito $(\mathrm{OR}=2,97$; IC95\%=1,41-6,46), cerca de 3 vezes mais, nos bebês cujas mães não possuíam instrução. Com relação ao tipo de hospital, observou-se nesta etapa analítica o efeito protetor do nascimento em hospital não público.

Em relação aos fatores de risco referentes aos determinantes intermediários, isto é, a atenção à saúde (número de consultas de pré-natal e tipo de parto), identificou-se que na consulta pré-natal, a OR apresentou-se decrescente conforme aumentava o número de consultas, assim, quanto maior o número de consultas de pré-natal menor as chances de óbito neonatal. $\mathrm{O}$ tipo de parto não esteve associado a um maior risco de óbito entre os RNs estudados. Os dados estão apresentados na Tabela 2.
Tabela 1 - Fatores de risco distais (não ajustados) para a mortalidade neonatal, município de Serra-ES, 2001-2005.

\begin{tabular}{l|c|c|c|c|c}
\hline \multirow{2}{*}{ Característica } & \multicolumn{2}{|c|}{ Óbito } & \multirow{2}{*}{ OR } & IC 95\% & P \\
\cline { 2 - 3 } & Sim & Não & & & \\
\hline Faixa etária (anos) & 5 & 217 & 2,97 & \\
$<15$ & 62 & 6.531 & 1,22 & 0,13 \\
15 a 19 & 176 & 22.700 & & \\
20 a 34 & 30 & 2.531 & 1,53 & \\
35 ou mais & & & & \\
\hline
\end{tabular}

Escolaridade materna (anos)

$\begin{array}{lrrr}\text { Nenhuma } & 9 & 382 & 3,01 \\ 1 \text { a } 3 & 22 & 2.442 & 1,15 \\ \text { 4 a } 7 & 103 & 11.643 & 1,13 \\ \text { 8 a } 11 & 109 & 13.732 & 1,02 \\ \geq 12 & 27 & 3.457 & \end{array}$

Tipo de hospital

SUS

$242 \quad 26.561 \quad 1,63 \quad 1,11-2,38 \quad 0,11$

Não-SUS

$30 \quad 5.369$

Tabela 2 - Fatores de risco intermediários (não ajustados) para a mortalidade neonatal, município de Serra-ES, 2001-2005.

\begin{tabular}{c|c|c|c|c|c}
\hline \multirow{2}{*}{ Características } & \multicolumn{2}{|c|}{ Óbito } & \multirow{2}{*}{ OR } & IC 95\% & p \\
\cline { 2 - 3 } & Sim & Não & & & \\
\hline Consultas pré-natal & & & & & \\
Nenhuma & 13 & 594 & 3,56 & $1,99-6,38$ & \\
$1-3$ & 38 & 2.672 & 2,32 & $1,59-3,36$ & $<0,001$ \\
$4-6$ & 106 & 11.034 & 1,56 & $1,19-2,05$ & \\
7 ou mais & 105 & 17.104 & & & \\
\hline
\end{tabular}

Tipo de parto

$\begin{array}{lllll}\text { Cesáreo } & 136 & 14.621 & 1,17 & 0,93-1,49 \\ \text { Vaginal } & 137 & 17.327 & & \end{array}$

0,181 
Tabela 3 - Fatores de risco proximais (não ajustados) para a mortalidade neonatal, município de Serra-ES, 2001-2005.

\begin{tabular}{l|l|l|l|l|l}
\hline \multirow{2}{*}{ Característica } & \multicolumn{2}{|c|}{ Óbito } & & & \\
\cline { 2 - 3 } & Sim & Não & & IC 95\% & P \\
\cline { 2 - 3 } & & & & \\
\hline
\end{tabular}

\section{Sexo do RN}

$\begin{array}{llllll}\text { Masculino } & 146 & 16.449 & 1,08 & 0,85-1,38 & 0,502 \\ \text { Feminino } & 127 & 15.528 & & \end{array}$

\section{Idade gestacional (semanas)}

$\begin{array}{lrrrrr}\leq 31 & 49 & 201 & 43,05 & 30,74-61,54 & \\ 32 \text { a } 36 & 52 & 1.659 & 5,59 & 4,08-7,66 & <0,0001 \\ \geq 37 & 168 & 29.974 & & & \end{array}$

\begin{tabular}{|c|c|c|c|c|c|}
\hline Peso (gramas) & & & & & \\
\hline$<1500$ & 62 & 308 & 37,73 & $27,54-51,69$ & \\
\hline $1.500-2.499 \mathrm{~g}$ & 54 & 2.218 & 4,46 & $3,34-6,24$ & $<0,0001$ \\
\hline$>2.500 \mathrm{~g}$ & 157 & 29.430 & & & \\
\hline
\end{tabular}

\section{Apgar $1^{\circ}$ minuto}

$\begin{array}{lrrrrr}\text { Gravemente anoxiado } & 54 & 346 & 29,3 & 21,10-40,68 & \\ \text { Anóxia neonatal moderada } & 46 & 1.515 & 5,7 & 4,08-7,96 & <0,0001 \\ & & & & & \\ \text { Boas condições de vitalidade } & 152 & 28.536 & & & \end{array}$

\section{Apgar $5^{\circ}$ minuto}

$\begin{array}{lccccc}\text { Gravemente anoxiado } & 23 & 109 & 32,62 & 20,36-52,25 & \\ \text { Anóxia neonatal moderada } & 34 & 215 & 24,44 & 16,58-36,04 & <0,000 \\ & & & & & \\ \text { Boas condições de vitalidade } & 195 & 30.145 & & & \end{array}$

Tipo de gestação

\begin{tabular}{|c|c|c|c|c|}
\hline Múltipla & 10 & 550 & 2,17 & $1,15-4,10$ \\
\hline Única & 262 & 31.706 & & \\
\hline
\end{tabular}

Ao avaliarmos as características proximais (sexo, idade gestacional, peso ao nascer, tipo de gestação, Apgar no $1^{\circ}$ e Apgar no $5^{\circ}$ minuto) e sua associação com a mortalidade neonatal, encontramos que apenas o sexo do RN não mostrou associação estatisticamente significante (Tabela 3). Desta forma, observou-se risco de óbito semelhante entre nascidos vivos do sexo masculino e feminino. Por outro lado, características como peso ao nascer, idade gestacional, índice de Apgar e tipo de gestação mostraram-se associadas ao aumento da mortalidade neonatal.

Verificou-se que a idade gestacional $\leq 31$ semanas $(O R=43,05)$ representou o fator de exposição com maior chance para o óbito neonatal. Observou-se também que, quanto menor o peso dos nascidos vivos maior é a chance de mortalidade neonatal: os nascidos vivos com peso inferior a $1.500 \mathrm{~g}$ apresentaram risco de morrer no período neonatal 37,73 vezes maiores. No entanto, entre os nascidos vivos com peso de 1.500 a $2.499 \mathrm{~g}$, o risco passa a ser 4,56 vezes maior do que os nascidos vivos com peso superior a $2.500 \mathrm{~g}$.

Os RNs cuja evolução do Apgar entre o primeiro e quinto minuto de vida revelou hipóxia grave (escore $<7$ ) apresentaram chance de morte superior aos que não sofreram hipóxia. Observou-se ainda, que o risco de morte em relação ao Apgar no $5^{\circ}$ minuto foi maior que no Apgar no $1^{\circ}$ minuto em todos os grupos estudados.

Quanto ao tipo de gestação, nota-se que, em gestações múltiplas, as chances de mortalidade neonatal são 2,17 vezes maiores quando comparadas com as gestações únicas.

Na tabela 4 estão descritos os resultados da regressão logística e as OR ajustadas nos três níveis de determinação. No nível distal, das três variáveis incluídas na regressão logística: mães sem instrução $(O R=2,44)$, idade materna < 15 anos $(\mathrm{OR}=2,89)$, idade materna $>35$ anos $(\mathrm{OR}=1,54)$ e nascer em hospital público (OR $=1,57)$ mostraram maiores chances para o óbito neonatal.

No nível intermediário, nenhuma consulta de pré-natal $(\mathrm{OR}=3,48)$ representou o fator de exposição com maior chance para o óbito.

Em relação ao nível proximal, todas as cinco variáveis incluídas no modelo mantiveram associação significante com a mortalidade neonatal, após ajuste pelas variáveis dos níveis intermediário e distal. Os RNs com peso < 1500g representaram o fator de exposição com maior chance de óbito $(O R=5,49)$, seguido em ordem decrescente pelo Apgar $<7$ no primeiro minuto de vida $(\mathrm{OR}=4,05)$, Apgar $<7$ no quinto minuto de vida $(\mathrm{OR}=3,81)$, idade gestacional $\leq 31$ semanas $(\mathrm{OR}=3,45)$ e gestação múltipla $(\mathrm{OR}=2,26)$. 
Tabela 4. Regressão logística hierarquizada dos fatores de risco da mortalidade neonatal, município de Serra-ES, 2001-2005.

\begin{tabular}{l|c|c|c|c}
\hline \multirow{2}{*}{ Nível } & \multicolumn{2}{|c|}{ Óbito } & \multirow{2}{*}{ OR } & \multirow{2}{*}{ IC 95\% } \\
\cline { 2 - 3 } & Sim & Não & & \\
\hline DISTAL
\end{tabular}

\begin{tabular}{lcccc}
\hline $\begin{array}{l}\text { Faixa etária (anos) } \\
<15\end{array}$ & 5 & 217 & 2,89 & $1,16-7,18$ \\
15 a 19 & 62 & 6.531 & 1,23 & $0,92-1,65$ \\
20 a 34 & 176 & 22.700 & & \\
$\quad$ 35 ou mais & 30 & 2.531 & 1,54 & $1,04-2,29$ \\
\hline $\begin{array}{l}\text { Escolaridade Materna } \\
\text { Nenhuma }\end{array}$ & 9 & 382 & 2,44 & $1,13-5,29$ \\
1 a 3 & 22 & 2.442 & 0,95 & $0,53-1,70$ \\
4 a7 & 103 & 11.643 & 0,94 & $0,60-1,47$ \\
8 a11 & 109 & 13.732 & 0,92 & $0,59-1,41$ \\
$\geq 12$ & 27 & 3.457 & & \\
\hline
\end{tabular}

\begin{tabular}{lcccc}
\hline Tipo de hospital & & & & \\
SUS & 242 & 26.561 & 1,57 & $1,05-2,33$ \\
Não SUS & 30 & 5.369 & & \\
\hline
\end{tabular}

\section{INTERMEDIÁRIO}

\begin{tabular}{lcccc}
\hline $\begin{array}{l}\text { Consultas pré-natal } \\
\text { Nenhuma }\end{array}$ & 13 & 594 & 3,48 & $1,86-6,49$ \\
1 a 3 & 38 & 2.672 & 2,39 & $1,62-3,53$ \\
4 a 6 & 106 & 11.034 & 1,62 & $1,62-1,08$ \\
7 ou mais & 105 & 17.104 & & \\
\hline
\end{tabular}

\begin{tabular}{ccccc}
\hline $\begin{array}{c}\text { Tipo de parto } \\
\text { Cesáreo }\end{array}$ & 136 & 14.621 & 1,52 & $1,17-1,97$ \\
Vaginal & 137 & 17.327 & & \\
\hline PROXIMAL & & & & \\
\hline
\end{tabular}

\section{PROXIMAL}

Idade gestacional (semanas)

31

32 a 36

$\geq 37$

Peso (gramas)

$<1500$

$1.500-2.499 \mathrm{~g}$

$>2.500 \mathrm{~g}$

\begin{tabular}{lcccc}
\hline Apgar $\mathbf{1}^{\mathbf{0}}$ minuto & & & & \\
$\quad$ Gravemente anoxiado & 54 & 346 & 4,05 & $2,10-7,79$ \\
$\quad$ Anóxia neonatal moderada & 46 & 1.515 & 2,82 & $1,90-4,18$ \\
$\quad$ Boas condições de vitalidade & 152 & 28.536 & & \\
\hline Apgar 50 minuto & & & & \\
$\quad$ Gravemente anoxiado & 23 & 109 & 3,81 & $1,71-8,49$ \\
$\quad$ Anóxia neonatal moderada & 34 & 215 & 2,99 & $1,58-5,66$ \\
$\quad$ Boas condições de vitalidade & 195 & 30.145 & & \\
\hline Tipo de gestação & & & & \\
$\quad \begin{array}{l}\text { Múltipla } \\
\text { Única }\end{array}$ & 10 & 550 & 2,26 & $1,03-4,96$ \\
\hline
\end{tabular}

\section{DISCUSSÃO}

Entre os determinantes distais, todas as variáveis socioeconômicas (idade materna, escolaridade materna e tipo de hospital de nascimento) analisadas estiveram associadas com a mortalidade neonatal no Município de Serra.

A idade materna é considerada uma variável importante na determinação da mortalidade neonatal, pois os extremos de idade para concepção apresentam maiores chances de complicações e consequente risco de morte.

Em estudo com dados sobre o município de Santo André-SP(5), verificou-se uma associação entre a idade da mãe e o baixo peso ao nascer. Observa-se uma proporção maior de crianças com baixo peso ao nascer de mães jovens (menos de 15 anos) do que em idade maior (mais de 35 anos).

Em estudo realizado nos municípios da Grande Vitória, no ano de 2007, ficou explícita a maior predisposição à prematuridade em recém-nascidos de mães com idade entre 10 a 14 anos, do que os demais ${ }^{(7)}$.

A escolaridade materna é considerada um indicador da posição social. O nível educacional da mãe pode ser compreendido como um fator relacionado ao perfil cultural e ao comportamento ligado aos cuidados de saúde, que têm um efeito importante na determinação da mortalidade ${ }^{(1)}$. Pesquisas demonstraram que, ao analisar a correlação entre a escolaridade materna e indicadores obstétricos, encontrou-se associações estatisticamente significativas entre menor escolaridade e ocorrência de baixo peso ao nascer; maior número de partos prematuros e menores números de consultas de pré-natal. Assim, concluiu-se que a escolaridade materna pode ser considerada um marcador obstétrico de risco para a gestante e o RN. Ressaltou-se ainda que a associação significativa de menor escolaridade com mortalidade neonatal pode ser decorrente da menor condição social e da dificuldade de acesso das mães aos serviços de saúde ${ }^{(8)}$.

No que tange a variável nascer no hospital, observou-se que a escolha do hospital, público ou privado, está relacionada ao nível socioeconômico das gestantes. Estudiosos verificaram na análise multivariada que os nascidos vivos em hospital público-estatal apresentaram um risco de morte no período neonatal 2,3 vezes superiores aos nascidos vivos em hospital privado(1). Verificou-se também associação entre nascimento em hospitais públicos conveniados ao Sistema Único de Saúde (SUS) e maior risco de mortalidade neonatal ${ }^{(9)}$.

Em relação aos determinantes intermediários (número de consultas de pré-natal e tipo de parto), um dos determinantes mais importantes para prevenir a mortalidade neonatal é a freqüência 
ao pré-natal, visto que, quanto maior o numero de consultas durante o período gestacional menor a chance de mortalidade neonatal, talvez por permitir detectar doenças maternas e fetais, bem como fazer intervenções precoces.

O aumento do número de consultas pré-natais possibilitou reduzir a prevalência de CIUR, a prematuridade, a número de nascidos vivos com baixo peso e de óbitos por afecções do período perinatal ${ }^{(11)}$.

Para consultas de pré-natal verificou-se que o risco de mortalidade foi de 2,32 maior no grupo de mães que haviam realizado até três consultas e chegou a 3,56 no grupo cujas mães não realizaram consulta. Autores corroboram tais resultados, ao constatar que mães com menos de 5 consultas apresentavam um risco 2,5 vezes maior do que as demais ${ }^{(12)}$.

Nesse estudo, o tipo de parto também demonstrou estar associado a um maior risco de óbito entre os RNs. Na literatura, verifica-se que o parto cesárea se apresenta como fator de proteção em recém-nascidos de baixo peso e/ou em prematuros ${ }^{(13)}$. No entanto, não é consenso seu efeito protetor para a mortalidade neonatal, pois outros autores não observaram diminuição de risco de óbito pós-cesárea(14-15) . Pesquisa na cidade do Recife-PE, realizada no período de 2000 a 2002, também não correlacionou tipo de parto com maior chance de óbito neonatal ${ }^{(6)}$.

Entre os determinantes proximais estudados, a prematuridade e o baixo peso ao nascer têm sido considerados, sistematicamente, causas importantes de mortalidade neonatal ${ }^{(3,16)}$. O peso ao nascer é considerado como uma das características mais importantes do $\mathrm{RN}$ e um dos principais fatores relacionados com a mortalidade neonatal; por isso é uma variável muito utilizada nos estudos epidemiológicos.

Os RN com baixo peso podem ser classificados em dois grupos fisiopatologicamente distintos: os prematuros e os bebês a termo com CIUR $^{(17)}$.

Os bebês com CIUR estão etiologicamente relacionados: o fator intrínseco (capacidade diminuída de transporte de nutrientes em nível placentário), a fator extrínseco (deficiência de nutrientes antes e ou durante a gravidez), ou a uma combinação dos dois. Entre os fatores de risco associados à ocorrência de bebês com CIUR estão incluídos: baixo nível de instrução materna, pré-natal inadequado, estado nutricional deficiente antes da gravidez, ganho de peso insuficiente durante a gestação, infecção geniturinária, tabagismo, ausência de cônjuge e primiparidade ${ }^{(18)}$.

Já em relação à prematuridade sabe-se que é um indicador de risco para mortalidade neonatal e está estreitamente relacionada com o baixo peso ao nascer. Sendo que as principais causas são: desnutrição, infecções, prematuridade iatrogênica, hipertensão, rotura prematura das membranas, entre outras ${ }^{(17)}$.

O Apgar tem sido considerado uma variável preditiva importante da mortalidade neonatal. Essa variável está mais diretamente relacionada à qualidade da assistência no momento do parto, apesar da influência das condições prévias do RN durante o período intra-uterino, que determinam sua vitalidade no momento do nascimento. Uma assistência inadequada durante a qual ocorra sofrimento fetal pode resultar em um RN com Apgar abaixo de oito, mesmo com gestação a termo e com peso adequado(17). Neste sentido, corroborando com nossos resultados, diversos autores ${ }^{(5,8,11)}$ identificaram o Apgar como variável associada a maior chance de óbito.

Quanto ao tipo de gestação é reconhecido que entre os nascidos de gravidez múltipla há uma alta prevalência de prematuridade e de baixo peso. Por essa razão, a gemelaridade é considerada um fator materno associado à mortalidade neonatal ${ }^{(1)}$. Em relação a essa variável, foi encontrado um risco 2,17 vezes maior de óbito entre os RNs de gestações múltiplas. Confirmando nossos resultados, pesquisadores identificaram associação entre gestação múltipla e maior risco para mortalidade neonatal ${ }^{(1,11)}$, sendo observado em estudos anteriores uma chance 5,95 vezes maior de óbito em RNs de gestação múltipla ${ }^{(1)}$.

Dentre as variáveis investigadas, o sexo foi a única não associada à mortalidade neonatal. Semelhante aos nossos resultados, outros estudos também não identificaram esta associação $0^{(1,2,6)}$.

Cabe considerar que algumas pesquisas verificaram associação entre sexo masculino e risco de morte ${ }^{(2,14,16)}$. Estudos afirmam que, em relação à mortalidade segundo o gênero, é comum observar que o coeficiente de mortalidade do sexo masculino é maior que o do feminino em todas as idades ${ }^{(19)}$.

Confirmando alguns de nossos achados, diversas pesquisas apontam que após realizarem a regressão logística ajustada, as variáveis: Apgar $<7$ no $1^{\circ}$ e $5^{\circ}$ minuto de vida, peso ao nascer e idade gestacional foram confirmadas como fatores de risco mais significativos para mortalidade neonatal ${ }^{(2,6,13,16)}$.

Embora os fatores de risco para o óbito neonatal sejam diversos e interajam entre si é necessária a implantação de políticas públicas para a promoção da saúde da mulher antes da concepção, durante a gestação e, da criança depois do parto, de maneira a gerar um desenvolvimento contínuo e integrado à saúde, com objetivo de garantir acesso aos serviços de qualidade. Esse avanço pode ser decisivo no combate a mortalidade neonatal.

Pode se destacar, que desde 2003 quando ocorreu à mudança para a Gestão Plena de Atenção Básica Ampliada, o município de Serra vem fazendo investimentos na área da saúde ampliando o atendimento e cobertura pré-natal, bem como estimulando o parto e o nascimento humanizado e melhorando a qualidade da assistência ao recém-nascido. Ao lado de iniciativas para melhoria da assistência pré-natal, do controle de nascimentos com baixo peso e a redução de causas específicas do pós-neonatal, diversas ações foram introduzidas com o propósito de reduzir a mortalidade como: campanhas de vacinação em massa, incentivo ao aleitamento materno, implantação do programa de agentes comunitários de saúde, treinamento dos profissionais de saúde nos programas do Ministério da Saúde, entre outras. Também, em 2005, a maternidade municipal de Serra recebeu o título de Hospital Amigo da Criança, pela UNICEF e pelo Ministério da Saúde, sendo o segundo hospital do Estado do Espírito Santo a recebê-lo ${ }^{(20)}$.

Quanto às limitações do estudo, o uso de dados secundários não foi um fator limitante, pois, esse estudo apresentou uma baixa perda de dados que pode estar relacionada à boa qualidade de preenchimento das DNs e DOs. Fica evidente o potencial de utilização do SIM para o monitoramento da qualidade da assistência materno-infantil, pois a investigação dos óbitos pode revelar os fatores de risco que levam os recém-nascidos a terem 
sua vida interrompida por causas perfeitamente evitáveis. Por outro lado, deve se atentar para a qualidade da informação, pois estudo utilizando o SIM para analisar a tendência da mortalidade perinatal no município de Belo Horizonte no período de 1984 a 2005, mostra que houve melhora na qualidade da informação apenas para o registro da escolaridade materna e peso ao nascer e aponta que esforços devem ser direcionados no sentido de melhorar a completude do SIM para variáveis importantes na elaboração dos indicadores perinatais(21).

Além disso, pesquisas que utilizam o procedimento de linkage de bancos de dados de nascidos vivos e óbitos infantis mostram a viabilidade e as vantagens dessa estratégia utilizando-se como fonte de dados os sistemas de informações em saúde disponíveis no Brasil. Essa estratégia de análise possibilita o monitoramento dos fatores de risco para mortalidade neonatal e das mudanças na magnitude desses fatores nas coortes de nascidos vivos.

\section{CONCLUSÃO}

Conclui-se que, após os ajustes na regressão logística, os fatores de risco associados à mortalidade neonatal no município de Serra foram: nenhuma escolaridade da mãe, idade materna $<15$ anos e > 35 anos, nascer em hospital público, nenhuma consulta de pré-natal, RNs com peso $<1500$, Apgar gravemente anoxiado $(<7)$ no primeiro e no quinto minuto de vida, idade gestacional $\leq 31$ semanas, e gestação múltipla.

Por fim, apesar dos fatores que levam ao óbito neonatal serem variados e interatuarem entre si com diferentes intensidades é necessária a adoção de medidas mais diretas nos serviços de saúde e também na comunidade, que garantam um atendimento adequado ao binômio mãe-filho desde o pré-natal, parto e em todo período neonatal. Este atendimento proporcionaria a detecção, em tempo hábil, de outros fatores de risco para morte neonatal como os relacionados aos nascimentos de pré-termos e a anóxia neonatal.

O estudo possibilitou o uso das bases de dados de nascimento (SINASC) e óbitos (SIM), recomendando essa estratégia para o monitoramento e identificação dos principais fatores de risco para a mortalidade neonatal e reafirmou a importância da relação dos fatores biológicos com o óbito. $\mathrm{E}$ também, fornece subsídios ao poder público para o enfrentamento dos fatores que levam a mortalidade neonatal.

\section{REFERÊNCIAS}

1. Morais Neto OL, Barros MB. Fatores de risco para mortalidade neonatal e pós-neonatal na Região Centro-Oeste do Brasil: "linkage" entre bancos de dados de nascidos vivos e óbitos infantis. Cad. Saúde Pública 2000;16(2):477-85.

2. Ribeiro AM, Guimarães MJ, Lima MC, Sarinho SW, Coutinho SB. Fatores de risco para mortalidade neonatal em Crianças com baixo peso ao nascer. Rev Saúde Pública 2009;43(2):246-55

3. Martins EF, Velasquez-Melendez G. Determinantes da mortalidade neonatal a partir de uma coorte de nascidos vivos, Montes Claros, Minas Gerais, 1997-1999. Rev Bras Saúde Mater Infant 2004;4(4):405-12.

4. Almeida MF, Mello Jorge MHP. O uso da técnica de "Linkage" de sistemas de informações em estudos de coorte sobre mortalidade neonatal. Rev Saúde Pública 1996;30(2):141-7.

5. Almeida MF. Mortalidade neonatal em Santo André. São Paulo. Tese [Doutorado em Enfermagem]- Departamento de Epidemiologia, Universidade de São Paulo; 1996.

6. Silva CFS, Leite AJM, Almeida NMJS, Gondim RC. Fatores de risco para mortalidade infantil em municípios do Nordeste do Brasil: Linkage entre bancos de dados de nascimentos vivos e óbitos infantis - 2000 a 2002. Rev Bras Epidemiol 2006;9(1):69-80.

7. Nader PRA, Cosme LA. Parto prematuro de adolescentes: influência de fatores sociodemográficos e reprodutivos, Espírito Santo, 2007. Esc Anna Nery Rev Enferm 2010;14(2):338-45.
8. Haiddar FH, Oliveira UF, Nascimento LFC. Escolaridade materna: correlação com os indicadores obstétricos. Cad Saúde Pública 2001;17(4):1025-9.

9. Almeida MF, Novaes HMD, Alencar GP, Rodrigues LC. Mortalidade neonatal no município de São Paulo: influências do peso ao nascer e de fatores sócio-demográficos e assistências. Rev Bras Epidemiol 2002;5(1):93-106.

10. Prefeitura Municipal de Serra [homepage na internet] Serra: perfil socioeconômico. Serra; 2009 [acesso em: 01 out 2010]. Disponível em: < http://www.serra.es.gov.br. >

11. Kilsztajn S, Rossbach A, Carmo MSN, Sugahara GTL. Assistência pré-natal, baixo peso e prematuridade no estado de São Paulo, 2000. Rev Saúde Pública 2003;37(3):303-10.

12. Victora CG. Intervenções para reduzir a mortalidade infantil pré-escolar e materna no Brasil. Rev Bras Epidemiol 2001;4(1):3-67.

13. Almeida SDM, Barros MBA. Atenção à saúde e mortalidade neonatal: estudo de caso-controle realizado em Campinas, SP. Rev Bras Epidemiol 2004;7(1):22-34.

14. Duarte JLMB, Mendonça GAS. Avaliação dos óbitos neonatais em recém-nascidos de muito baixo peso em quatro maternidades no município do Rio de Janeiro. Cad Saúde Pública 2005;21(2):387-95.

15. Helena ETS, Sousa CA, Silva CA. Fatores de risco para mortalidade neonatal em Blumenau, Santa Catarina: linkage entre bancos de dados. Rev Bras Saúde Mater Infant 2005;5(2):209-217. 
16. Macharelli CA, Oliveira LR. Perfil do risco de óbito de crianças menores de um ano residentes em localidade do Estado de São Paulo, Brasil, 1987. Rev Saúde Pública 1991;25(2):121-8.

17. D'Orsi E, Carvalho MS. Perfil de nascimentos no município do Rio de Janeiro: uma análise espacial. Cad Saúde Publica 1998;14(2):367-79.

18. Serafin D. Mortalidade neonatal em Maringá. São Paulo. Tese. [Doutorado em Enfermagem]- Faculdade de Saúde Pública, Universidade de São Paulo; 2002.
19. Laurenti R. Mortalidade infantil nos Estados Unidos, Suécia e Estado de São Paulo. Rev Saúde Pública 1987;21(3):268-73.

20. Lima EFA. Fatores associados à mortalidade neonatal no município de Serra, ES (2001-2005). Rio de Janeiro. Dissertação [Mestrado em Enfermagem]- Escola de Enfermagem Anna Nery, Universidade Federal do Rio de Janeiro; 2007.

21. Martins EF, Lana FCF, Maria E. Tendência da mortalidade perinatal em Belo Horizonte, 1984 a 2005. Rev Bras Enferm 2010;63(3):446-51. 